\title{
Role of intranasal fentanyl in breakthrough pain management in cancer patients
}

This article was published in the following Dove Press journal:

Cancer Management and Research

25 September 2010

Number of times this article has been viewed

\section{Wojciech Leppert}

Chair and Department of Palliative Medicine, Poznan University of Medical Sciences, Poznan, Poland
Correspondence: Wojciech Leppert Chair and Department of Palliative Medicine, Poznan University of Medical Sciences, Osiedle Rusa 25A, 6I-245 Poznan, Poland

Tel +48 61 8738303

Fax +48 6I 8738303

Email wojciechleppert@wp.pl

\begin{abstract}
Fentanyl is a strong opioid analgesic, which is commonly used in the form of a transdermal patch for the treatment of chronic cancer pain. An intranasal route of fentanyl administration is a novel treatment for breakthrough cancer pain (BTCP). The prevalence, assessment, and management of BTCP is outlined in this paper, and basic pharmacodynamic and pharmacokinetic properties, dosing guidelines, and clinical experience with the use of intranasal fentanyl in this indication are discussed. Intranasal fentanyl is an attractive and convenient mode of BTCP treatment in opioid-tolerant patients due to its quick onset and short duration of action, noninvasive administration route, high bioavailability, and avoidance of a hepatic first-pass effect. Until now, few clinical trials have been conducted with intranasal fentanyl, but all have confirmed its usefulness and acceptability in BTCP treatment. Intranasal fentanyl may be used in opioid-tolerant patients without nasal pathologies. The dose should be titrated in each patient regardless of the regular opioid dose administered. Future studies should compare intranasal fentanyl with other fentanyl formulations used for BTCP management, and with analgesia, adverse effects, and quality of life taken into consideration.
\end{abstract}

Keywords: adverse effects, analgesia, breakthrough pain, intranasal fentanyl, opioid analgesics, treatment

\section{Introduction}

In patients with cancer pain of moderate to severe intensity, treatment is based on regular administration of opioid analgesics and adjuvant analgesics (coanalgesics) for effective pain relief. However, in spite of this regular treatment, an exacerbation of pain may appear, and is known as "breakthrough" pain. Breakthrough cancer pain (BTCP) is defined as a transitory exacerbation of pain to greater than moderate intensity, which occurs on a baseline pain of moderate intensity or less in a patient receiving opioid therapy. ${ }^{1}$ The term "breakthrough" pain was coined by Hilgier in Poland. ${ }^{2}$

\section{Prevalence and assessment of BTCP}

The prevalence of BTCP in patients admitted to a hospice was assessed in a prospective survey. Among 414 consecutive admissions, 33 patients were confused and too unwell to take part in the study, 136 patients were pain-free, and 245 patients reported 404 pains (range $1-5$ per patient). Of the latter patients, 218 (89\%) had BTCP and identified 361 pains (range 1-5 per patient). BTCP was classified as somatic (46\%), visceral $(30 \%)$, neuropathic $(10 \%)$, or of mixed etiology $(16 \%)$. Thirty-eight percent of pains were severe or excruciating. The average number of breakthrough pain episodes was 7 (range 1-14), and 49\% of pain episodes occurred 
suddenly. Most (59\%) were unpredictable and 72\% lasted for more than 30 minutes. Among the patients surveyed, $75 \%$ were dissatisfied with their pain relief. ${ }^{3}$

In another observational cross-sectional study conducted in different settings (home and outpatient/inpatient units), the prevalence of BTCP in Catalonia, Spain, was determined in oncology patients treated by palliative care teams. BTCP was reported by 163 (41\%) of 387 patients in this study. A total of 244 episodes (mean 1.5 episodes/patient/day) of mean intensity $7.3 \pm 2.0$ compared with $2.9 \pm 2.7$ for persistent pain (both 0-10 scales) was reported. Morphine was used to treat $52 \%$ of the episodes, while $25 \%$ were untreated. ${ }^{4}$

In a prospective study of 63 cancer patients who reported moderate pain or less for more than 12 hours daily and had received stable opioid dosing for a minimum of two consecutive days, 41 (64\%) reported severe or excruciating BTCP. Fiftyone different pains were described (median four pains per day, range 1-3600). Twenty-two (41\%) pains were paroxysmal in onset, and the remainder was of more gradual onset. Duration of pain varied from seconds to hours (median 30 minutes, range 1-240 minutes). Fifteen (29\%) of the pains were related to the fixed opioid dose, occurring solely at the end of the dosing interval (currently not classified as BTCP). Twenty-eight (55\%) of the pains were precipitated and, of these, 22 were caused by an action of the patient (incident pain) and six were associated with a nonvolitional precipitant. The pathophysiology of the pain was believed to be somatic in $17(33 \%)$, visceral in 10 (20\%), neuropathic in $14(27 \%)$, and mixed in $10(20 \%)$ of cases. Pain was related to the tumor in $42(82 \%)$, the effects of therapy in seven (14\%), and neither in two (4\%) cases. ${ }^{1}$

In a cross-sectional survey, inpatients with cancer completed several instruments to assess pain and mood (Memorial Pain Assessment Card), pain-related interference with function (Brief Pain Inventory, BPI), depressed mood (Beck Depression Inventory), and anxiety (Beck Anxiety Inventory). Of 178 eligible patients, $164(92.2 \%)$ met the criteria for controlled baseline pain. The median age was 50.6 (range 26-77) years. Seventy-five percent of the patients had metastases and the majority had mixed nociceptive-neuropathic pain. The median Karnofsky score was 60 (range 40-90). Eighty-four (51.2\%) patients had experienced BTCP during the previous day. The median number of episodes was six (range 1-60) and the median interval from onset to peak was three minutes (range one second to 60 minutes). Two-thirds of patients $(61.7 \%)$ could identify precipitants (movement $20.4 \%$, end-of-dose failure $13.2 \%$ ), and pain was unpredictable in most cases (78.2\%). Patients with BTCP had more intense $(P<0.001)$ and more frequent $(P<0.01)$ background pain than patients without BTCP. Greater pain-related functional impairment was also associated with BTCP (BPI, $P<0.001$ ), as well as worse mood (visual analog scale, $P<0.05$, Beck Depression Inventory, $P<0.001)$ and greater anxiety (Beck Anxiety Inventory, $P<0.001$ ). Multivariate analysis confirmed that BTCP independently contributed to impaired functioning and psychologic distress. ${ }^{5}$

In a prospective cross-sectional study, 1095 patients with cancer pain were evaluated by 58 clinicians from 24 countries using patient-rated items from the BPI and observer-rated measures, including demographic and tumor-related data, occurrence of BTCP, and responses on checklists for pain syndromes and pathophysiologies. The clinicians reported BTCP in $64.8 \%$ of patients. Physicians from English-speaking countries were significantly more likely to report BTCP than physicians from other countries. BTCP was associated with higher pain scores and functional interference on the BPI. Multivariate analysis showed an independent association of BTCP with the presence of more than one pain, a vertebral pain syndrome, pain due to plexopathy, and an English-speaking country. ${ }^{6}$

Assessment of BTCP is crucial to its appropriate and effective management. The etiology, characteristics, and mechanisms of BTCP should be elucidated. ${ }^{7}$ The temporal course, severity, and impact of BTCP on the patient's daily activities and quality of life should be assessed, as well as the psychologic and social burden of BTCP. A detailed history and thorough clinical examination should be performed. Assessment of background pain relief is also a very important step in effective management of BTCP. ${ }^{8}$ Assessment of BTCP is similar to the evaluation of baseline pain because no specific instrument for BTCP assessment exists. BTCP evaluation is based on a detailed history

Table I Questions asked for the assessment of breakthrough pain. Copyright @ 2009. Adapted with permission from Davies AN, Dickman A, Reid C, Stevens AM, Zeppetella G. The management of cancer-related breakthrough pain: Recommendations of a Task Group of the Science Committee of the Association for Palliative Medicine of Great Britain and Ireland. Eur J Pain. 2009;|3:33|-338
I. Onset of pain
2. Frequency of pain
3. Site of pain
4. Radiation of pain
5. Quality (character of pain)
6. Intensity (severity) of pain
7. Duration of pain
8. Exacerbating factors
9. Relieving factors
10. Response to analgesics
II. Response to other interventions
12. Associated symptoms
13. Interference with daily living. 
(Table 1), ${ }^{9}$ thorough clinical examination, and, if doubts exist as to the cause of pain, radiologic imaging may be helpful. ${ }^{10}$

\section{Management of BTCP}

Several expert recommendations have addressed the issue of guidelines for BTCP management. ${ }^{11-13}$ Generally, there are limited data based on randomized controlled studies, so only general recommendations have been proposed for the management of BTCP (Table 2).

BTCP may be classified into three subtypes, ie, nonincident pain, incident pain, and end-of-dose failure. The latter cannot be considered as BTCP because it is associated with an insufficient dose or too long an interval between administration of analgesia. The management of end-of-dose pain is usually to increase the dose or to shorten the interval of regular analgesic administration to control the baseline pain. Nonincident pain is called spontaneous ("idiopathic") pain, where pain episodes are not related to an identified precipitant, and so are unpredictable in nature. ${ }^{14}$ Incident pain is due to specific triggers, such as walking or movement in bed, and generally it is predictable (volitional incident pain). Predictable incident pain is best managed prophylactically. Incident pain associated with smooth muscle contraction or

Table 2 Recommendations for the management of cancer-related breakthrough pain. Copyright (C) 2009. Adapted with permission from Davies AN, Dickman A, Reid C, Stevens AM, Zeppetella G. The management of cancer-related breakthrough pain: Recommendations of a Task Group of the Science Committee of the Association for Palliative Medicine of Great Britain and Ireland. Eur J Pain. 2009; | 3:33 |-338

I. Patients with pain should be assessed for the presence of BTCP

2. Patients with BTCP should have the pain specifically assessed

3. The management of breakthrough cancer pain should be individualized

4. Consideration should be given to treatment of underlying cause of the pain

5. Consideration should be given to avoidance/treatment of precipitating factors of pain

6. Consideration should be given to modification of the background analgesic regimen/around the clock medication

7. Opioids are the rescue medication of choice in the management of breakthrough cancer pain episodes

8. The dose of opioid rescue medication should be determined by individual titration

9. Nonpharmacologic methods may be useful in the management of breakthrough cancer pain episodes

10. Nonopioid analgesics may be useful in the management of breakthrough cancer pain episodes

II. Interventional techniques may be useful in the management of breakthrough cancer pain

12. Patients with breakthrough cancer pain should have this pain specifically re-assessed coughing is more difficult to predict (nonvolitional incident pain). ${ }^{12}$

The management of BTCP is based on the use of shortacting formulations of opioids such as morphine, oxycodone, hydromorphone, buprenorphine, and methadone (rescue dose). In a prospective study of hospice inpatients with BTCP, the times to onset of pain relief after different rescue opioid analgesic administration were compared. Patients presented with, on average, 1.7 different types of breakthrough pain (range 1-4). The average number of breakthrough pains was four per day (range 1-8), and the average duration was 35 minutes (range 15-60), with most occurring suddenly and unpredictably. Patients used morphine, oxycodone, hydromorphone, methadone, or oral transmucosal fentanyl citrate as rescue medication, and the average time to meaningful pain relief following administration of analgesia was 31 (range 5-75) minutes. No difference was found between morphine, oxycodone, and hydromorphone. Methadone appeared to work faster than morphine $(P<0.01)$, but no faster than oxycodone or hydromorphone, whereas oral transmucosal fentanyl citrate worked faster than morphine, oxycodone, hydromorphone, and methadone $(P<0.001) .{ }^{15}$ In spite of a delayed analgesic effect of these drugs, their advantages include a huge clinical experience and ease of administration, usually by the oral route, which is especially useful for patients treated at home. However, the slow onset of analgesia (20-30 minutes) and delayed peak analgesia (60-90 minutes) often results in ineffective analgesia with a prolonged duration of effect (3-6 hours). ${ }^{12}$

Apart from the convenient oral route, other routes may be used to administer opioid analgesics for the treatment of BTCP. The intravenous route is very effective, although is used mostly in the hospital setting. ${ }^{16}$ The subcutaneous route of opioid administration is effective and may also be used at home. ${ }^{17}$ Rectal administration may be considered, especially in a liquid formulation which seems to have a shorter peak effect. ${ }^{18}$ However, this route may be inconvenient for many patients. Intrathecal administration of opioids, local anesthetics, and clonidine may also be considered. ${ }^{19}$ In some patients, treatment with nonopioid analgesics, such as paracetamol, ${ }^{20}$ nonsteroidal anti-inflammatory drugs, ${ }^{21}$ and bisphosphonates may be effective in incident bone pain. ${ }^{22}$ Ketamine in neuropathic or mixed neuropathic and bone pain syndromes may be administered. ${ }^{23}$ Severe BTCP not responding to high doses of intravenous morphine may be treated successfully with an intrathecal bolus of levobupivacaine or sublingual ketamine ${ }^{24}$ Radiotherapy may be beneficial in bone pain. ${ }^{25}$ Surgical and anesthetic techniques should be considered in patients with specific pain syndromes. ${ }^{26}$ 


\section{Fentanyl in BTCP}

A more recent method of BTCP management is use of rapid-onset opioids, including different formulations of fentanyl, ie, oral transmucosal fentanyl citrate, buccal fentanyl tablets, and intranasal fentanyl. The advantages of these new formulations are their quicker onset and shorter duration of action, which both fit the time profile of BTCP better because pain usually starts and intensifies quickly (in approximately three minutes) and lasts 30-60 minutes. However, limited data are available on the efficacy and tolerability of these newer preparations, and health professionals have limited familiarity with their use. The high cost of these new therapies should also be carefully considered. ${ }^{14}$

Fentanyl, a fenylpiperidine derivative, is a synthetic opioid analgesic, the chemical structure of which is similar to that of pethidine, and it has been used since $1959 .{ }^{27}$ Fentanyl is a pure $\mu$ opioid receptor agonist, and is approximately 100 -fold more potent than morphine. ${ }^{28}$ Because of its high lipid solubility, fentanyl is distributed in all human tissues and, in contrast with morphine, crosses the bloodbrain barrier easily and quickly, which explains its strong analgesic effect and lower intensity of the common adverse opioid effects associated with a central (nausea and vomiting) and peripheral (constipation) mode of action. ${ }^{29,30}$ The drug is characterized by a short duration of analgesia (3060 minutes) after intravenous administration. In contrast with morphine, fentanyl does not induce histamine release. Fentanyl may induce chest wall rigidity, so transdermal fentanyl patches are not recommended for the treatment of patients with dyspnea, ${ }^{31,32}$ who are usually treated with morphine. ${ }^{33}$ However, several published case series have reported successful use of oral transmucosal fentanyl citrate $^{34}$ or intranasal fentanyl in the treatment of breathlessness..$^{35}$

Fentanyl is metabolized predominantly in the liver but also in the duodenum (at a mean rate which is approximately half that of hepatic metabolism) through cytochrome (CYP)3A4 to pharmacologically inactive metabolites (predominantly norfentanyl and hydroxyfentanyl), which are excreted in the urine. ${ }^{36}$ Because many drugs inhibit CYP3A4, and the enzyme activity differs significantly between individuals, numerous pharmacokinetic drug interactions are possible with fentanyl. ${ }^{37}$ Following intravenous administration, approximately $85 \%$ of fentanyl is found predominantly as metabolites in the urine within 72 hours. In patients with renal or liver failure, the fentanyl dose should be adjusted due to the possibility of accumulation of fentanyl and its metabolites. ${ }^{38}$ Careful fentanyl dosing is recommended in older patients, who are usually more susceptible to the effects of opioid analgesics due to changes in protein binding ability, and in the distribution and elimination of drugs.

\section{Intranasal fentanyl}

Intranasal drugs should be administered in small volumes to avoid runoff into the pharynx. In adults, the nasal cavity volume is $15-20 \mathrm{~mL}$ and the surface area is $150-180 \mathrm{~cm}^{2}$. A single administration volume in one nostril to avoid runoff of the drug to the pharynx is $150 \mu \mathrm{L}$. Different vehicles and additives may be required to obtain a therapeutically effective dose in a small-volume solution, and some of these act as penetration enhancers. ${ }^{39}$

The high lipid solubility of fentanyl may play an important role in its good absorption through the nasal mucosa. For the intranasal route of fentanyl administration, the time to maximal plasma concentration is 13 minutes and the bioavailability is $70 \%-90 \% .^{40} \mathrm{As}$ a result of intranasal administration, the drug bypasses the liver, thereby avoiding the hepatic first-pass effect. ${ }^{41}$ The concomitant use of nasal mucosal decongestants should be avoided because these agents may decrease fentanyl bioavailability. ${ }^{42}$ In a single-dose, randomized, crossover, double-blind study conducted in healthy volunteers, a fentanyl dose of $0.054 \mathrm{mg} / 1.08 \mathrm{~mL}$ was administered to both nostrils. Time to peak concentration was five minutes after drug administration and the peak level was $0.29 \pm 0.076 \mathrm{ng} / \mathrm{mL}$, with a bioavailability of $71 \%{ }^{43}$ The pharmacokinetics of intranasal fentanyl spray were assessed in a randomized, open-label, two-period, crossover trial conducted in 19 patients with BTCP. ${ }^{44}$ Intranasal fentanyl spray was administered as a single dose in one nostril. Each dose was separated by at least 48 hours. Mean fentanyl plasma concentrations increased in a dose-dependent manner, peaking for all doses at 9-15 minutes after intranasal fentanyl spray administration. Median times to peak concentration were 15,12 , and 15 minutes for the 50,100, and $200 \mu \mathrm{g}$ doses of intranasal fentanyl spray, respectively. Mean ( \pm standard deviation) values for peak concentrations were $351( \pm 226), 595( \pm 400)$, and $1195( \pm 700)$ $\mathrm{pg} / \mathrm{mL}$, respectively, indicating dose proportionality. Six patients $(31.6 \%)$ experienced adverse events during the treatment period, the majority being mild in severity. Intranasal fentanyl appears to work faster than oral transmucosal fentanyl citrate or buccal fentanyl tablets, and the intranasal route may be used in patients who suffer from dry mouth and therefore cannot use either oral transmucosal fentanyl citrate or buccal fentanyl tablets. ${ }^{45}$ 


\section{Intranasal fentanyl in postoperative pain}

Several studies have been conducted in patients with postoperative pain and have established the efficacy and safety of nasal fentanyl administration in both adults ${ }^{46,47}$ and in children. ${ }^{48,49}$ In a randomized, double-blind study, Striebel et al compared intranasal fentanyl spray with intravenous fentanyl in patients with pain following surgery for lumbar intervertebral disc protrusion. ${ }^{46}$ Twenty-two patients received six sprays of fentanyl $0.027 \mathrm{mg}$ intranasally and placebo intravenously, and 20 patients received placebo intranasally and fentanyl $0.027 \mathrm{mg}$ intravenously. Before the beginning of opioid titration and then every 10 minutes for at least one hour, pain was evaluated using a 101-point numeric rating scale and a verbal rating scale. In both groups, the doses were repeated every five minutes until patients were free of pain or refused further analgesics. All patients were satisfied with the pain relief achieved. The total fentanyl dose was 0.073 (range $0.027-0.162$ ) $\mathrm{mg}$ in the intravenous group and 0.11 (range 0.027-0.243) $\mathrm{mg}$ in the intranasal group. The onset of action after intranasal fentanyl was nearly as rapid as that after intravenous titration. In both groups, pain intensity significantly decreased within 10 minutes of drug administration, and pain reduction was comparable in both groups. Only at the 10,20, and 30-minute measurement points was the pain intensity significantly lower in the intravenous than in the intranasal group. One patient in the intravenous group showed a decrease in arterial hemoglobin oxygen saturation to less than $90 \%$. No other serious adverse effects were observed, and adverse events were similar in both patient groups and did not require cessation of treatment. Local adverse effects in the nasal cavity were not observed.

In a prospective, randomized, double-blind, doubledummy study, Toussaint et al compared fentanyl given as a $25 \mu \mathrm{g}$ intranasal bolus and $17.5 \mu \mathrm{g}$ for intravenous patientcontrolled analgesia with a lockout interval of six minutes in 48 patients on the day of surgery (orthopedic, abdominal, or thyroid). ${ }^{47}$ The first requested dose was doubled in both groups. Pain intensity and analgesia were assessed by a 101-point numeric rating scale and, together with vital parameters, were measured at 11 time points over 240 minutes. Onset of analgesia and first reduction in pain intensity on the numeric rating scale occurred at $21 \pm 11$ (range 15-45) minutes in the intranasal group and at $22 \pm 16$ (range 15-90) minutes in the intravenous patient-controlled analgesia group. Pain intensity was reduced from $55 \pm 11$ to $11 \pm 10$ in the intranasal group and from $53 \pm 8$ to $11 \pm 6$ in the intravenous patient- controlled analgesia group. Vital parameters remained stable and adverse effects were comparable in both groups. A judgment of "excellent" or "good" pain relief was given by 21 of 23 patients treated intranasally and 24 of 25 patients treated intravenously.

\section{Intranasal fentanyl in BTCP}

A few clinical studies have assessed the effects of intranasal fentanyl in the treatment of BTCP. Zeppetella reported two patients treated with nebulized and intranasal fentanyl for BTCP. Both patients had good pain relief without significant adverse effects. ${ }^{50}$ Zeppetella conducted a preliminary study with intranasal fentanyl citrate in cancer patients with nociceptive pain. ${ }^{51}$ Patients enrolled stayed at the inpatient unit and were treated with regularly administered opioids, ie, morphine in 11 patients and transdermal fentanyl in one patient. The rescue drug was a short-acting oral morphine preparation. A single dose of intranasal fentanyl citrate $20 \mu \mathrm{g}$ was used, with no possibility of dose titration. Of 12 patients enrolled, eight reported good or very good pain relief. Nine patients continued treatment with intranasal fentanyl citrate, including one patient who did not experience pain relief after drug administration. Treatment failure was observed in patients receiving higher equivalent morphine daily doses (120 mg or more) whereas responders to intranasal fentanyl citrate received lower morphine doses. The treatment was well tolerated with no significant systemic adverse effects. Nasal itching was observed in two patients which disappeared with repeated drug application.

Kress et al investigated the efficacy and long-term tolerability of intranasal fentanyl spray 50-200 $\mu \mathrm{g}$ in the treatment of BTCP in opioid-tolerant patients in a doubleblind, randomized, placebo-controlled, crossover trial. ${ }^{52}$ Patients were recruited from pain centers, anesthesiology departments, palliative care units, and oncology clinics in Austria, Denmark, France, Germany, and Poland. Eligible patients were adults with cancer receiving a stable dose of long-term opioid treatment for the control of background pain. Patients were treated at home with their effective dose of intranasal fentanyl spray $(50,100$, or $200 \mu \mathrm{g})$ or placebo in a randomized sequence for three weeks, followed by a 10-month, open-label tolerability phase during which they received their effective dose of intranasal fentanyl spray. Patients were allowed to use their usual rescue medications. Pain was assessed on an 11-point numeric rating scale $(0=$ no pain, $10=$ worst pain imaginable $)$ with pain intensity difference (PID) at 10 minutes after drug administration. A total of 120 patients were enrolled and achieved an effective dose; 113 were randomized and 111 
were included in the intent-to-treat analysis set (intranasal fentanyl spray $50 \mu \mathrm{g} 18$ patients, $100 \mu \mathrm{g} 48$ patients, and $200 \mu \mathrm{g} 45$ patients, and placebo 110 patients). The PID at 10 minutes after intranasal fentanyl spray administration was two-fold greater than that after placebo $(P<0.001)$. The mean response rate with all three doses of intranasal fentanyl spray was $51.1 \%$ versus $20.9 \%$ with placebo. The prevalence of adverse effects was 22/111 (19.8\%) during the efficacy period, and the most frequently reported adverse effects were nausea in five patients $(4.5 \%)$ and vertigo in two $(1.8 \%)$. No serious adverse effects were considered to be related to the study medication. In all, 108 patients entered the extension period, with a mean duration of exposure to intranasal fentanyl spray of 134.9 days. The most common adverse event reported during this period was progression of underlying malignant disease in 55 (50.9\%) patients, which was not considered to be treatment-related.

Mercadante et al compared the efficacy of intranasal fentanyl spray with that of oral transmucosal fentanyl citrate for the relief of BTCP in an open-label, crossover trial. ${ }^{53}$ Adult cancer patients receiving stable background opioid treatment and experiencing BTCP episodes were recruited from 44 study centers in seven European countries (Austria, France, Germany, Italy, Poland, Spain, and the UK). Of the 196 patients enrolled, 139 were randomized to receive intranasal fentanyl spray, followed by oral transmucosal fentanyl citrate, or vice versa. Patients were titrated to an effective dose of one agent (intranasal fentanyl spray 50, 100, or $200 \mu \mathrm{g}$, or oral transmucosal fentanyl citrate 200, 400, 600, 800, 1200, or $1600 \mu \mathrm{g}$ ) to treat six BTCP episodes, then titration and treatment were repeated with the other agent. The primary outcome was patient-recorded time to onset of meaningful pain relief. Secondary outcomes included PID at 10 and 30 minutes, sum of PID at 15 and 60 minutes, ease of administration, treatment preference, and relationship between background opioid dose and effective intranasal fentanyl spray dose.

Additional outcome measures included the proportions of episodes with $\geq 33 \%$ and $\geq 50 \%$ pain intensity reduction and PID at additional time points. Among the intention-to-treat population $(\mathrm{n}=139)$, the median time to onset of meaningful pain relief was 11 minutes for intranasal fentanyl spray versus 16 minutes for oral transmucosal fentanyl citrate; $65.7 \%$ of patients had a faster time to meaningful pain relief onset with intranasal fentanyl spray $(P<0.001)$. PID was significantly greater for intranasal fentanyl spray than for oral transmucosal fentanyl citrate from five minutes postdosing. Significantly more intranasal fentanyl spray-treated BTCP episodes achieved clinically important pain relief $(\geq 33 \%$ versus $\geq 50 \%$ pain intensity reduction) up to 30 minutes postdosing. The proportions of episodes treated with intranasal fentanyl spray and oral transmucosal fentanyl citrate achieving a pain intensity reduction of $\geq 33 \%$ at five minutes were $25.3 \%$ versus $6.6 \%(P<0.001$, respectively $)$, and at 10 minutes were $51.0 \%$ versus $23.6 \%(P<0.001)$. The proportions of episodes treated with intranasal fentanyl spray and oral transmucosal fentanyl citrate achieving $\geq 50 \%$ pain intensity reduction at five minutes were $12.8 \%$ versus $2.1 \%(P<0.001)$, and at 10 minutes were $36.9 \%$ versus $9.7 \%(P<0.001)$, respectively. Higher sum of PID scores at 15 and 60 minutes were achieved with intranasal fentanyl spray $(P<0.001)$. More patients preferred intranasal fentanyl spray to oral transmucosal fentanyl citrate $(P<0.001)$, and more patients found it easy or very easy to use. Both treatments were well tolerated. In the safety population analysis $(n=139), 56.8 \%(n=79)$ of patients experienced more than one adverse event during the trial. The only adverse effect that occurred in $5 \%$ or more patients in either treatment group was nausea. Among those patients who experienced serious adverse events $(13.7 \%, \mathrm{n}=19)$, none were considered to be related to either study medication. There was a weak correlation between effective intranasal fentanyl spray doses and background opioid doses.

Stam et al evaluated the cost effectiveness of intranasal fentanyl spray and oral transmucosal fentanyl citrate for the treatment of BTCP. ${ }^{54}$ A decision-analytic model was developed to estimate the costs and benefits associated with intranasal fentanyl spray and oral transmucosal fentanyl citrate. The model translated expected reduction in pain of BTCP episodes into resource use/cost savings and into quality of life gains for an assumed remaining life expectancy of six months. Efficacy data were obtained from clinical trials and indirectly compared, adjusting for differences in placebo responses. With intranasal fentanyl spray, $63 \%$ of BTCP (95\% confidence interval [CI] 51\%-70\%) was avoided, which was greater than with oral transmucosal fentanyl citrate $(29 \%$, CI $17 \%-42 \%$ ). Given the short life expectancy of these patients, this efficacy difference translated into a 0.055 gain in qualityadjusted life years with intranasal fentanyl spray relative to oral transmucosal fentanyl citrate. Due to its greater efficacy, intranasal fentanyl spray is expected to reduce medical resource use and bring about greater cost savings than oral transmucosal fentanyl citrate. Despite the uncertainty of resource data, there is a greater than $99 \%$ probability that intranasal fentanyl spray is cost-effective relative to oral transmucosal fentanyl citrate.

Vissers et al undertook an extended meta-analysis of six randomized, controlled trials that compared intranasal fentanyl 
spray with oral transmucosal fentanyl citrate, buccal fentanyl tablets, and oral morphine for the treatment of BTCP. ${ }^{45}$ The endpoint was PID reported on a 10-point numeric rating scale up to 60 minutes after intake. Intranasal fentanyl spray provided the greatest reduction in pain relative to placebo, ie, PID was 1.7 points (95\% CI 1.4-1.9) at 15 minutes, $2.0(1.6-2.3)$ at 30 minutes, $2.0(1.5-2.4)$ at 45 minutes, and $1.9(1.5-2.4)$ at 60 minutes. PIDs for oral transmucosal fentanyl citrate and buccal fentanyl tablets relative to placebo were $0.4(0.0-0.8)$ and $0.5(0.3-0.7)$, respectively, at 15 minutes. Both treatments provided a reduction in pain superior to placebo at other time points. Intranasal fentanyl spray displayed a more than $99 \%$ probability of providing the greatest pain reduction out of all interventions at 15 minutes after intake. This was maintained for any measured time point before 45 minutes when compared with buccal fentanyl tablets, and for any measured time point before 60 minutes when compared with oral transmucosal fentanyl citrate. Only from 45 minutes onwards did oral morphine show a greater pain reduction than placebo.

\section{Conclusion}

Intranasal fentanyl is a promising option for the treatment of patients with BTCP, in spite of concerns of possible abuse, mostly in patients with chronic nonmalignant pain. ${ }^{55}$ It provides a convenient mode of breakthrough pain treatment in opioid-tolerant cancer patients due to a quick onset and short duration of action, which matches well the breakthrough pain pattern in most cancer patients with moderate to severe pain. The dose of intranasal fentanyl should be titrated regardless of the regular opioid dose administered. The advantages of intranasal fentanyl are its noninvasive administration route, high bioavailability with avoidance of an hepatic first-pass effect and high patient acceptability. In contrast with oral transmucosal fentanyl citrate and buccal fentanyl tablets, intranasal fentanyl may be recommended in the treatment of BTCP in patients with dry mouth. Although only a few clinical trials have been conducted with intranasal fentanyl in BTCP, all have confirmed its usefulness, and a meta-analysis has found that it works faster than oral transmucosal fentanyl citrate, buccal fentanyl tablets, or oral morphine. ${ }^{45}$ Intranasal fentanyl may be used in opioid-tolerant patients without nasal pathologies. Potential pharmacokinetic and pharmacodynamic drug interactions should be kept in mind when using this new mode of fentanyl delivery. The results of a preliminary study indicate the possibility of effective intranasal ketamine use in BTCP. ${ }^{56}$ Future studies should compare intranasal fentanyl with other fentanyl formulations for BTCP, evaluating analgesia, adverse effects, and quality of life.

\section{Disclosure}

The author reports no conflict of interest in this work.

\section{References}

1. Portenoy RK, Hagen NA. Breakthrough pain: Definition, prevalence and characteristics. Pain. 1990;41:273-281.

2. Hilgier M. Bole przebijajace w chorobie nowotworowej. Wspolcz Onkol. 2001;5:168-174.

3. Zeppetella G, O'Doherty CA, Collins S. Prevalence and characteristics of breakthrough pain in cancer patients admitted to a hospice. J Pain Symptom Manage. 2000;20:87-92.

4. Gómez-Batiste X, Madrid F, Moreno F, et al. Breakthrough cancer pain: Prevalence and characteristics in patients in Catalonia, Spain. $J$ Pain Symptom Manage. 2000;20:45-52.

5. Portenoy RK, Payne D, Jacobsen P. Breakthrough pain: Characteristics and impact in patients with cancer pain. Pain. 1999;81:129-134.

6. Caraceni A, Martini C, Zecca E, Portenoy RK; Working Group of an IASP Task Force on Cancer Pain. Breakthrough pain characteristics and syndromes in patients with cancer pain. An international survey. Palliat Med. 2004;18:177-183.

7. Mercadante S. Characteristics and mechanisms of BTCP. Eur J Palliat Care. 2010;17:8-12.

8. Davies AN. Assessment of BTCP. Eur J Palliat Care. 2010;17:13-15.

9. Foley KM. Acute and chronic cancer pain syndromes. In: Doyle D, Hanks G, Cherny N, Calman K, editors. Oxford Textbook of Palliative Medicine. Oxford: Oxford University Press; 2004.

10. Leppert W. Kliniczna ocena bolu w chorobie nowotworowej. Twoj Mag Med. 2005;5:34-40.

11. Mercadante S, Radbruch L, Caraceni A, et al; Steering Committee of the European Association for Palliative Care (EAPC) Research Network. Episodic (Breakthrough) Pain. Consensus Conference of an Expert Working Group of the European Association for Palliative Care. Cancer. 2002;94:832-839.

12. Davies AN, Dickman A, Reid C, Stevens AM, Zeppetella G. The management of cancer-related breakthrough pain: Recommendations of a Task Group of the Science Committee of the Association for Palliative Medicine of Great Britain and Ireland. Eur J Pain. 2009;13: 331-338.

13. Hanks GW, de Conno F, Cherny N, et al; Expert Working Group of the Research Network of the European Association for Palliative Care. Morphine and alternative opioids in cancer pain: The EAPC recommendations. Br J Cancer. 2001;84:587-593.

14. Hui D, Bruera E. Breakthrough pain in cancer patients: The need for evidence. Eur J Palliat Care. 2010;17:58-67.

15. Zeppetella G. Opioids for cancer breakthrough pain: A pilot study reporting patient assessment of time to meaningful pain relief. $J$ Pain Symptom Manage. 2008;35:563-567.

16. Mercadante S, Villari P, Ferrera P, Bianchi M, Casuccio A. Safety and effectiveness of intravenous morphine for episodic (breakthrough) pain using a fixed ratio of with the oral daily morphine dose. J Pain Symptom Manage. 2004;27:352-359.

17. Enting R, Mucchiano C, Oldenmenger WH, et al. The "pain pen" for breakthrough cancer pain: A promising treatment. J Pain Symptom Manage. 2005;29:213-217.

18. de Conno, Ripamonti C, Saita L, et al. Role of rectal route in treating cancer pain: A randomized crossover trial of oral versus rectal morphine administration in opioid-naive cancer patients with pain. J Clin Oncol. 1995;13:1004-1008.

19. Hassenbusch SJ, Portenoy RK, Cousins M, et al. Polyanalgesic consensus conference 2003: An update on the management of pain by intraspinal drug delivery - report of an expert panel. J Pain Symptom Manage. 2004;27:540-563.

20. Davies AN, Vriens J, Kennett A, McTaggart M. An observational study of oncology patients' utilisation of breakthrough pain medication. J Pain Symptom Manage. 2008;35:406-411. 
21. Portenoy RK. Adjuvant analgesic agents. Hematol Oncol Clin North Am. 1996;10:103-119.

22. Fulfaro F, Casuccio A, Ticozzi C, Ripamonti C. Pamidronate in incident pain due to bone metastases. J Pain Symptom Manage. 2001;22: 630-631.

23. Leppert $\mathrm{W}$. The role of ketamine in the management of neuropathic cancer pain - a Polish experience. In: Wells CD, editor. Proceedings of the Third International Congress on Neuropathic Pain. Athens: Medimond International; 2010.

24. Mercadante S, Arcuri E, Ferrera P, Villari P, Mangione S. Alternative treatments of breakthrough pain in patients receiving spinal analgesics for pain. J Pain Symptom Manage. 2005;30:485-491.

25. Leppert W, Nowakowska E. Rola radioterapii w leczeniu objawow zaawansowanej choroby nowotworowej. Med Paliat Prakt. 2008;2: 33-47.

26. Burton AW, Reddy SK, Shah HN, Tremont-Lukats I, Mendel E. Percutaneous vertebroplasty - a technique to treat refractory spinal pain in the setting of advanced metastatic cancer: A case series. J Pain Symptom Manage. 2005;30:87-95.

27. Moser KM. Transdermal fentanyl in cancer pain. Am Fam Phys. 1992; 45:2289-2294.

28. Prommer E. The role of fentanyl in cancer-related pain. $J$ Palliat Med. 2009;12:947-954.

29. Ahmedzai S, Brooks D. Transdermal fentanyl versus sustained-release oral morphine in cancer pain: Preference, efficacy, and quality of life. J Pain Symptom Manage. 1997;13:254-261.

30. Leppert W, Luczak J, Gorzelinska L, Kozikowska J, Wozniak SP. The use of transdermal fentanyl (Durogesic) in the treatment of cancer pain. J Pain Symptom Manage. 2000;20:S79.

31. Gumulka WS. Narkotyczne leki przeciwbolowe. In: Gumulka W, Meszaros J, editors. Wspolczesne Metody Zwalczania Bolu. Warszawa: Osrodek Informacji Naukowej Polfa; 2000.

32. Zylicz Z, van Rijn-van der Plaat LL. Fentanyl may increase breathlessness in a patient with motor neuron disease. J Pain Symptom Manage. 2006;32:199-200.

33. Thomas JR, von Gunten CF. Management of dyspnoea. J Support Oncol. 2003;1:23-34.

34. Gauna AA, Kang SK, Triano ML, Swatko ER, Vanston VJ. Oral transmucosal fentanyl citrate for dyspnea in terminally ill patients: An observational study. J Palliat Med. 2008;11:643-648.

35. Sitte T. Nasal application of fentanyl citrate as symptom control against breathlessness in palliative care - overview and case report. Wien Med Wochenschr. 2009;159:566-570. German.

36. Zech DFJ, Lehmann KA, Grond S. Transdermal/TTS/fentanyl in cancer pain management. Progr Palliat Care. 1994;2:37-42.

37. Labroo RB, Paine MF, Thummel KE, Kharasch ED. Fentanyl metabolism by human hepatic and intestinal cytochrome P4503A4: Implications for interindividual variability in disposition, efficacy, and drug interactions. Drug Metab Dispos. 1997;25:1072-1080.

38. Murtagh FE, Chai MO, Donohoe P, Edmonds PM, Higginson IJ. The use of opioid analgesia in end-stage renal disease patients managed without dialysis: Recommendations for practice. $J$ Pain Palliat Care Pharmacother. 2007;21:5-16.

39. Dale O, Hjortkjaer R, Kharasch ED. Nasal administration of opioids for pain management in adults. Acta Anaesthesiol Scand. 2002;46: 759-770.

Cancer Management and Research

\section{Publish your work in this journal}

Cancer Management and Research is an international, peer-reviewed open access journal focusing on cancer research and the optimal use of preventative and integrated treatment interventions to achieve improved outcomes, enhanced survival and quality of life for the cancer patient. The journal welcomes original research, clinical \& epidemiological

Submit your manuscript here: http://www.dovepress.com/cancer-management-and-research-journa
40. Anonymous. Bol przebijajacy w chorobie nowotworowej. Niedoceniany problem. Nowa metoda leczenia. Instanyl - fentanyl aerozol donosowy. Warszawa: Nycomed Pharma; 2009.

41. Foster D, Upton R, Christrup L, et al. Pharmacokinetics and pharmacodynamics of intranasal versus intravenous fentanyl in patients with pain after oral surgery. Ann Pharmacother. 2008;42:1380-1387.

42. Anonymous. Instanyl intranasal fentanyl spray. NYCOMED. Available at: www.instanyl.com. Accessed $2010 \mathrm{Jul} 9$.

43. Striebel HW, Kramer J, Luhmann I, Rohierse-Hohler I, Rieger A. Pharmakokinetische studie zur intranasalen gabe von fentanyl. Der Schmerz. 1993;7:122-125.

44. Kaasa S, Moksnes K, Nolte T, Lefebvre-Kuntz D, Popper L, Kress HG. Pharmacokinetics of intranasal fentanyl spray in patients with cancer and breakthrough pain. J Opioid Manage. 2010;6:17-26.

45. Vissers D, Stam W, Nolte T, Lenre M, Jansen J. Efficacy of intranasal fentanyl spray versus other opioids for breakthrough pain in cancer. Curr Med Res Opin. 2010;26:1037-1045.

46. Striebel HW, Koenigs D, Krämer J. Postoperative pain management by intranasal demand-adapted fentanyl titration. Anesthesiology. 1992;77: 281-285.

47. Toussaint S, Maidl J, Schwagmaier R, Striebel HW. Patient-controlled intranasal analgesia: Effective alternative to intravenous PCA for postoperative pain relief. Can J Anesth. 2000;47:299-302.

48. Galinkin JL, Fazi LM, Cuy RM, et al. Use of intranasal fentanyl in children undergoing myringotomy and tube placement during halothane and sevoflurane anesthesia. Anesthesiology. 2000;93:1378-1383.

49. Manjushree R, Lahiri A, Ghosh BR, Laha A, Handa K. Intranasal fentanyl provides adequate postoperative analgesia in pediatric patients. Can J Anesth. 2002;49:190-193.

50. Zeppetella G. Nebulized and intranasal fentanyl in the management of cancer-related breakthrough pain. Palliat Med. 2000;14:57-58.

51. Zeppetella G. An assessment of the safety, efficacy, and acceptability of intranasal fentanyl citrate in the management of cancer-related breakthrough pain: A pilot study. J Pain Symptom Manage. 2000;20: 253-258.

52. Kress HG, Oronska A, Kaczmarek Z, Kaasa S, Colberg T, Nolte T. Efficacy and tolerability of intranasal fentanyl spray 50 to $200 \mu \mathrm{g}$ for breakthrough pain in patients with cancer: A Phase III, multinational, randomized, double-blind, placebo-controlled, crossover trial with a 10-month, open-label extension treatment period. Clin Ther. 2009;31: $1177-1191$.

53. Mercadante S, Radbruch L, Davies A, et al. A comparison of intranasal fentanyl spray with oral transmucosal fentanyl citrate for the treatment of breakthrough cancer pain: An open-label, randomised, crossover trial. Curr Med Res Opin. 2009;25:2805-2815.

54. Stam W, Vissers D, Lenre M, et al. An economic evaluation of fastacting opioids for the treatment of breakthrough pain in cancer patients in Sweden. Eur J Pain. 2009;13 Suppl 1:S241.

55. Burkey A, Ballantyne J. The role of rapid-onset fentanyl in clinical practice. Eur J Palliat Care. 2010;17:88-90.

56. Carr DB, Goudas 1C, Denman WT, et al. Safety and efficacy of intranasal ketamine for the treatment of breakthrough pain in patients with chronic pain: A randomized, double-blind, placebo-controlled, crossover study. Pain. 2004;108:17-27.

studies, reviews \& evaluations, guidelines, expert opinion \& commentary, case reports \& extended reports. The manuscript management system is completely online and includes a very quick and fair peerreview system, which is all easy to use. Visit http://www.dovepress.com/ testimonials.php to read real quotes from published authors. 